\title{
A phase II study of tivozanib in patients with metastatic and nonresectable soft-tissue sarcomas
}

\author{
M. Agulnik ${ }^{*}$, R. L. B. Costa ${ }^{1}$, M. Milhem², A. W. Rademaker ${ }^{1}$, B. C. Prunder ${ }^{3}$, D. Daniels ${ }^{3}$, B. T. Rhodes ${ }^{3}$, \\ C. Humphreys ${ }^{1}$, S. Abbinanti ${ }^{1}$, L. Nye ${ }^{1}$, R. Cehic ${ }^{1}$, A. Polish ${ }^{1}$, C. Vintilescu' ${ }^{1}$, T. McFarland ${ }^{4}$, K. Skubitz $^{5}$, \\ S. Robinson ${ }^{6}$, S. Okuno ${ }^{6}$ \& B. A. Van Tine ${ }^{3}$ \\ ${ }^{1}$ Division of Hematology/Oncology, Northwestern University, Feinberg School of Medicine, Chicago; ${ }^{2}$ Division of Hematology/Oncology, University of lowa \\ Hospitals and Clinics, lowa City; ${ }^{3}$ Division of Hematology/Oncology, Washington University in St. Louis, St Louis; ${ }^{4}$ Division of Hematology/Oncology, University of \\ Wisconsin, Madison; ${ }^{5}$ Division of Hematology/Oncology, University of Minnesota, Minneapolis; ${ }^{6}$ Division of Hematology/Oncology, Mayo Clinic, Rochester, USA \\ 'Prior presentations: Presented in part at the 2015 American Society of Clinical Oncology Annual Meeting, Chicago, IL, USA. \\ *Correspondence to: Dr Mark Agulnik, Division of Hematology/Oncology, Northwestern University, 676 North Saint Claire Street, Suite 850, Chicago, IL 60610, USA. Tel: 1- \\ 312- 695-1222; Fax: 1-312-695-6189; \\ E-mail: m-agulnik@northwestern.edu
}

\begin{abstract}
Background: Soft tissue sarcomas (STSS) overexpress vascular endothelial growth factors (VEGF) and VEGF-receptors (VEGFR) activation have been associated with tumor aggressiveness. Tivozanib is a potent small molecule tyrosine kinase inhibitor against VEGFR1-3, with activity against PDGFR $\alpha / \beta$ and CKIT. The primary endpoint of this study was progression free survival (PFS) rate at 16 weeks. Secondary end points were overall survival (OS), response rate, safety and correlative studies.

Patients and methods: A Simon two-stage phase II trial was performed using tivozanib given orally at 1.5 mg daily, 3 week on 1 week off on a 28 day cycle until disease progression or intolerable toxicity.

Results: Fifty-eight patients were enrolled and treated with tivozanib. Leiomyosarcoma was the most common STS histological type in our cohort (47\%) and 27 patients (46\%) had received at least 3 lines of therapy prior to study entry. Up to 24 patients (41\%) had prior VEGF targeted therapies. Partial response and stable disease were observed in 2 (3.6\%) and 30 (54.5\%) patients. The 16 week PFS rate was 36.4\% [95\% confidence interval (CI) 23.7-49.1] and a median PFS of 3.5 months (95\% Cl 1.8-3). Median OS observed was 12.2 months (95\% Cl 8.1-16.8). The most frequent all grade toxicities were fatigue (48.3\%), hypertension (43.1\%), nausea (31\%) and diarrhea (27.6\%). The most common grade three toxicity was hypertension (22.4\%). Correlative studies demonstrate no correlation between the expression of VEGFR 1, 2 or 3, PDGFR $\alpha / \beta$ or FGF, and activity of tivozanib.
\end{abstract}

Conclusion: Tivozanib was well tolerated and showed antitumor activity with a promising median PFS and PFS rate at 4 months in a heavily pretreated population of metastatic STSs. Our results support further studies to assess the clinical efficacy of tivozanib in STS.

Clinical Trial Number: NCT01782313

Key words: sarcoma, phase II, tivozanib, tyrosine kinase inhibitor

\section{Introduction}

Soft tissue sarcomas (STSs) are malignant tumors of mesenchymal origin with a wide range of pathological and clinical behaviors. Approximately 15000 cases of soft tissue and bone sarcomas are diagnosed annually in the United States, accounting for approximately $1 \%$ of all malignancies [1]. For patients presenting with advanced nonresectable or metastatic sarcoma, palliation is the main objective of treatment. The prognosis remains dismal for patients with STS treated with doxorubicin alone or in combination with median overall survival ranging from 12 to 16 months [2-5]. Given lack of durable efficacy of chemotherapy for patients with advanced and metastatic disease, clinical trials are considered standard of care for the treatment of STS.

Angiogenesis plays an important role in the control of cancer progression and metastasis $[6,7]$. STSs are known to overexpress 
vascular endothelial growth factor (VGEF) and VEGF-receptors (VEGFR) 1 and $2[8,9]$. VEGFR pathway activation has been associated with tumor aggressiveness and chemotherapy resistance in STSs [10-12].

Pazopanib is a tyrosine kinase inhibitor (TKI), which targets VEGFR 1-3 and platelet derived growth factor receptor alpha and beta (PDGFR $\alpha / \beta)$, among many others [13]. The PALLETTE trial met its primary endpoint for progression free survival (PFS) improvement in patients with metastatic nonadipocytic STSs. [14] This randomized phase III placebo controlled study led to Food and Drug Administration approval of pazopanib for nonadipocytic and non-GIST STSs.

Tivozanib is potent oral TKI, which abrogates the VEGF pathway through inhibition of VEGFR1-3 signaling $[15,16]$. Other established targets of Tivozanib include PDGFR $\alpha / \beta$ and cKIT, albeit at higher concentrations. All targets of this kinase inhibitor have not been clearly identified. Phase I studies demonstrated that tivozanib is well tolerated at dose and schedule of $1.5 \mathrm{mg}$ po daily for 3 weeks followed by 1 week break in 28 day cycles [ 17 , 18]. Herein we report the results of a phase II open label study, which aims to evaluate the antitumor activity and tolerability of tivozanib in patients with metastatic and nonresectable STSs.

\section{Patients and methods}

\section{Patients}

The study population consisted of patients with metastatic, locally advanced or locally recurrent STS. The patients must have had a minimum of 1 and a maximum of 4 prior therapeutic regimens for recurrent/metastatic disease measurable by Response Evaluation Criteria in Solid Tumors (RECIST) 1.1.[19] Other inclusion criteria included age $\geq 18$ years, ECOG performance status $<2$, preserved marrow and organ function including absolute neutrophil count $\geq 1.5 \times 10^{9} / 1$, platelets $\geq 75 \times 10^{9} / 1$, total bilirubin $\leq 1.5 \times$ upper limit of normal (ULN), AST/ALT $\leq 2.5 \times$ ULN, creatinine $\leq 1.5 \times$ ULN, ejection fraction $>50 \%$ or above the institutional limit of normal. Exclusion criteria included uncontrolled hypertension (systolic blood pressure of $\geq 140 \mathrm{mmHg}$ or diastolic blood pressure of $\geq 90 \mathrm{mmHg}$ ), and electrocardiogram showing corrected QT interval (QTc) $>480$ ms using Bazett's formula (QT Interval/ $/$ RR interval). The following sarcoma histological subtypes were excluded from the study: alveolar soft-part sarcoma, chondrosarcoma, dermatofibrosarcoma, Ewing sarcoma, GIST, Kaposi sarcoma, mixed mesodermal tumor/carcinosarcoma, osteosarcoma, and other low-grade (grade 1) sarcomas, including well differentiated liposarcoma. Patients with active bleeding or clinically significant gastrointestinal (GI) abnormalities that may increase the risk for GI bleeding were ineligible for participation.

\section{Study design and treatment}

The study was an open label single arm phase II clinical trial based on Simon Optimal two-stage design [20]. It was conducted in agreement with the Declaration of Helsinki and the International Conference on Harmonization of Good Clinical Practice guidelines. The study protocol was approved by the institutional review board for each site, and all patients provided written informed consent for their participation. This trial was conducted through the Midwest Sarcoma Trials Partnership (MSTP) and funded by Aveo pharmaceutics. All patients were treated with tivozanib $1.5 \mathrm{mg}$ oral daily for 21 days followed by 7 days off $($ cycle $=28$ days). Treatment was continued until disease progression, unacceptable toxicity, or until discontinuation per patient preference or physician recommendation.

\section{Assessment of efficacy and adverse effects}

The primary end point of this study was to determine PFS rate at 16 weeks to assess the efficacy of tivozanib in advanced and metastatic STS. PFS was defined as the duration of time measured in months from start of treatment to death or progressive disease (PD). CT scans were performed every 2 cycles ( 8 weeks). In addition to a baseline scan, confirmatory scans were obtained a minimum of 4 weeks following initial documentation of objective response. Tumor response, stability and progression assessments were evaluated in this study using the RECIST criteria, version 1.1 [19]. Safety and drug related toxicities were assessed every 4 weeks at scheduled outpatient visits and at the end of study. The incidence, nature and severity of adverse events (AEs) were determined according to the National Cancer Institute Common Terminology Criteria for Adverse Events (NCICTCAE) version 4. All patients who receive at least one dose of tivozanib were evaluated for this endpoint. Secondary endpoints were overall survival (OS) measured from the first dose of therapy until death from any cause; overall response rate (ORR) defined as sum of partial response (PR) and complete response (CR) rates. The clinical benefit rate (CBR) defined as the ORR summed to the stable disease (SD) rate was also assessed. Patients who completed less than 2 weeks of treatment were replaced in the final analyses and were not evaluable for response. Patients for whom tissue slides and serum samples were available were included in the correlative analysis.

\section{Correlative studies}

Five micron sections of pretreatment archival tumor tissue were evaluated for VEGFR1, VEGFR2, VEGFR3, PDGFR $\alpha$ and PDGFR $\beta$ expression by standard immunohistochemistry. Peripheral blood was collected at baseline and at 30 days of treatment to assess for circulating levels of VEGF, PDGF and FGF2 by standard Elisa measurements.

\section{Immunohistochemistry}

The VEGFR1 (PA5-32408, Thermo Scientific Fredrick, MD, USA) VEGFR2 (PA5-16487 Thermo Scientific Fredrick, MD, USA), VEGFR3 (PA1-37712, Thermo Scientific Fredrick, MD, USA), PDGFR $\alpha$ (7HCLC, Thermo Scientific Fredrick, MD, USA) and PDGFR $\beta(06-495$, Millipore, Temecula, CA, USA) antibodies were used to determine protein expression using standard immunohistochemical (IHC) immunoperoxidase protocols. Slides were blindly assessed by two investigators that were blind to the specific antibody and the clinical data. Slides were scored on a $0-3+$ standard scoring scale with 0 being negative, +1 being $1-25 \%$ staining, +2 being $26-50 \%$ staining and +3 being $50 \%$ or greater staining. Kaplan-Meier analysis was then performed using MedCalc Statistical Software version 15.11.1 (MedCalc Software bvba, Ostend, Belgium; https://www.medcalc.org; 2015).

\section{Elisa analysis}

Total VEGF (ENZ-KOT156-001 (Enzo, Farmingdale, NY USA), PDGRFalpha (EHPDGFRBA, Thermo Scientific, Fredrick, MD, USA), PDGFRbeta (EHPDGFRB, Thermo Scientific, Fredrick, MD, USA) and FGF2 (AB999-FGF Basic (FGF2), Cambridge, UK) were measured by Elisa from serum using by manufactures protocol. The difference between day 30 and day 1 was plotted against days on trial and a linear regression was performed using MedCalc Statistical Software version 15.11.1 (MedCalc Software bvba, Ostend, Belgium; https://www.medcalc.org; 2015).

\section{Statistical analyses}

Based on historical controls, a PFS of $>40 \%$ at 4 months is considered promising for a second line STS therapy, and a 4 month PFS of $<20 \%$ is considered not promising [21]. The type I error was set at 0.05 and type II error was set at 0.10 . If 5 or more of the first 19 patients were alive and progression-free at 4 months, the trial would continue accrual to a total of 54 evaluable patients. After full accrual, 16 or more patients alive and 


\begin{tabular}{lcc} 
Table 1. Demographics and disease characteristics of enrolled patients \\
Characteristic & Patients $(\boldsymbol{n}=\mathbf{5 8})$ \\
\cline { 2 - 3 } & $\boldsymbol{n}$ & $\%$ \\
\hline Sex & & \\
Male & 24 & 41.4 \\
Female & 34 & 58.6 \\
Age, years & & \\
Median & 56.5 & - \\
Range & $21-82$ & \\
ECOG & & 72.4 \\
0 & 42 & 27.6 \\
1 & 16 & \\
Histology & & 46.6 \\
Leiomyosarcoma & 27 & 8.6 \\
MPNST & 5 & 6.9 \\
Liposarcoma & 4 & 5.2 \\
Angiosarcoma & 3 & 32.8 \\
Other & 19 & \\
Number of prior treatments & & 34.5 \\
1 & 20 & 19.0 \\
2 & 11 & 22.4 \\
3 & 13 & 24.1 \\
4 & 14 & \\
Prior anti-VEGF therapy & 24 & \\
\hline
\end{tabular}

VEGF, endothelial growth factors and receptors; MPNST, malignant peripheral nerve sheath tumor.

progression-free at 4 months would indicate a true 4 month PFS of $40 \%$ or more. The probability of stopping the study early is $67 \%$ under the null hypothesis.

With a 4 month PFS of $40 \%$, the median PFS may be expected to be 3.6 months. A sample of 54 patients evaluable for response would have $80 \%$ power to detect a median progression-free survival of 2.3 months versus 3.6 months assuming a one tailed test and a Type I error rate of $5 \%$. Time to event variables (PFS and OS) distributions was estimated using Kaplan-Meier curves. All other patients will be evaluable for toxicity only.

\section{Results}

\section{Patients and characteristics}

From February 2013 until January 2015, 58 patients were enrolled in the study and 55 were evaluable for response to treatment. Three patients were excluded from the efficacy analysis due to withdrawal of tivozanib within 14 days (stroke, gastrointestinal hemorrhage and posterior reversible encephalopathy syndrome). Forty-two patients had preserved PS (ECOG 0) and 27 had received at least 3 lines of prior treatment for metastatic or advanced STSs. Demographics and tumor characteristics are described in Table 1. A median number of 3 cycles (range 0-28) of tivozanib was administered.

\section{Responses, PFS and OS}

Seven of the first 19 patients were alive and progression-free at 4 months allowing expansion of accrual to 58 patients. With a

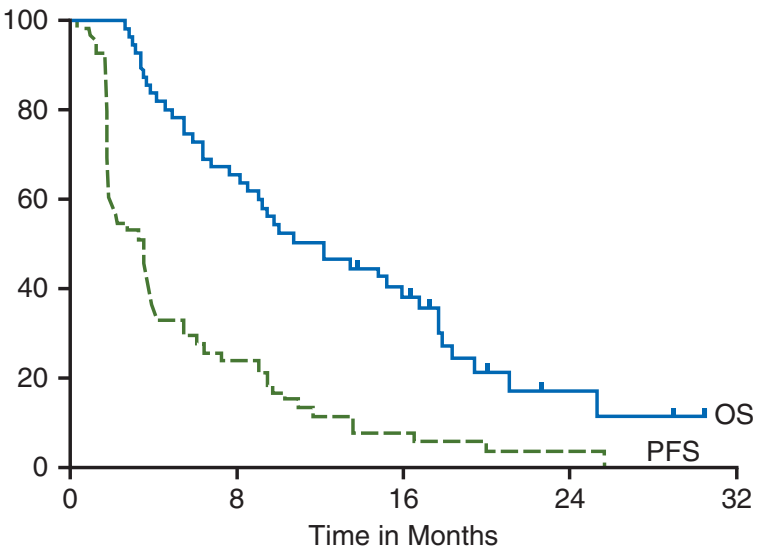

Figure 1. Kaplan-Meier curve of progression free survival (-) and overall survival (-) for all evaluable patients on study $(n=58)$. Median progression free survival was 3.5 months, (95\% Cl 1.8-3), and the median OS was 12.2 months, (95\% Cl 8.1-16.8).

median follow-up time of 9.6 months (range 0.9-30.5), of the 55 patients evaluable for progression, 19 patients were alive and progression free at 4 months with a 4 -month PFS rate of $36.4 \%$ (95\% CI 23.7-49.1). The endpoint to declare the true rate of being alive and progression-free at 4 months to be at least $40 \%$, as given by the Simon design, was met. The estimated median PFS was 3.5 months, (95\% CI 1.8-3). The median OS was 12.2 months, (95\% CI 8.1-16.8) (Figure 1).

PR and SD were observed in $2(3.6 \%)$ and $30(54.5 \%)$ patients with clinical benefit seen in 32 participants $(58.2 \%)$. The two partial responses were observed in patients with leiomyosarcoma and MPNST. Stable disease was seen in 14 patients with leiomyosarcoma, 3 with liposarcoma, 2 with synovial sarcoma, 2 with pleomorphic; the remaining 9 patients with stable disease were distributed among other histological subtypes. No CR was appreciated. SD $\geq 48$ weeks was achieved in 7 patients. Study participants with history of prior treatment with anti-VEGF pathway therapy (bevacizumab, pazopanib, sorafenib and/or sunitinib) had a 16 week PFS rate of $38.1 \%$ compared to $35.3 \%$ among antiVEGF naive participants $(P=0.98)$. Response was not related to number of prior treatments (12/19 PR/SD [63.2\%) with 1 prior treatment versus 20/36 (55.6\%) with 2-4 prior treatments, $P=0.77$ by Fisher's exact test $]$.

\section{Safety}

Tivozanib was well tolerated with four patients discontinuing treatment due to toxicity (posterior reversible encephalopathy syndrome, reduced ejection fraction, elevation of alkaline phosphatase, and fatigue). There were no grade five toxicities. The most frequent all grade toxicities were fatigue, hypertension, nausea and diarrhea (Table 2). The most common treatment related grade 3 events were hypertension, decreased ejection fraction, and fatigue. Overall grade 3, AEs were observed in 25 patients (43\%) treated with tivozanib. With the exception of hypertension, all grade 3 AEs had a frequency of the less than $10 \%$ of the cohort (Table 3 ).

\section{Correlatives}

To determine if there was a correlation between response to tivozanib and VEGFR expression, we performed IHC analysis 
Table 2. Frequency of treatment-related adverse events $\geq 10 \%$

\begin{tabular}{|c|c|c|c|c|c|c|}
\hline \multirow[t]{3}{*}{ Adverse event } & \multicolumn{6}{|c|}{ Toxicity grade } \\
\hline & \multicolumn{2}{|c|}{$1-2$} & \multicolumn{2}{|c|}{$3-4$} & \multicolumn{2}{|c|}{ Total } \\
\hline & $N$ & $\%$ & $N$ & $\%$ & $N$ & $\%$ \\
\hline Fatigue & 24 & 41.4 & 4 & 6.9 & 28 & 48.3 \\
\hline Hypertension & 11 & 19 & 14 & 24.1 & 25 & 43.1 \\
\hline Nausea & 18 & 31 & 0 & 0.0 & 18 & 31.0 \\
\hline Diarrhea & 16 & 27.6 & 0 & 0.0 & 16 & 27.6 \\
\hline Headache & 13 & 22.4 & 0 & 0.0 & 13 & 22.4 \\
\hline Hyperglycemia & 12 & 20.7 & 0 & 0.0 & 12 & 20.7 \\
\hline Anorexia & 12 & 20.7 & 0 & 0.0 & 12 & 20.7 \\
\hline Lymphopenia & 10 & 17.2 & 1 & 1.7 & 11 & 19.0 \\
\hline Hoarseness & 11 & 19.0 & 0 & 0.0 & 11 & 19.0 \\
\hline Aspartate aminotransferase increased & 9 & 15.5 & 1 & 1.7 & 10 & 17.2 \\
\hline GGT increased & 10 & 17.2 & 0 & 0.0 & 10 & 17.2 \\
\hline Vomiting & 9 & 15.5 & 0 & 0.0 & 9 & 15.5 \\
\hline Dyspnea & 9 & 15.5 & 0 & 0.0 & 9 & 15.5 \\
\hline Cough & 9 & 15.5 & 0 & 0.0 & 9 & 15.5 \\
\hline Alkaline phosphatase increased & 8 & 13.8 & 1 & 1.7 & 9 & 15.5 \\
\hline Myalgia & 8 & 13.8 & 1 & 1.7 & 9 & 15.5 \\
\hline Constipation & 8 & 13.8 & 0 & 0.0 & 8 & 13.8 \\
\hline Thrombocytopenia & 7 & 12.1 & 1 & 1.7 & 8 & 13.8 \\
\hline Dyspnea & 8 & 13.8 & 0 & 0.0 & 8 & 13.8 \\
\hline Weight loss & 6 & 10.3 & 1 & 1.7 & 7 & 12.1 \\
\hline Oral mucositis & 6 & 10.3 & 0 & 0.0 & 6 & 10.3 \\
\hline Hyponatremia & 6 & 10.3 & 0 & 0.0 & 6 & 10.3 \\
\hline
\end{tabular}

GGT, $\gamma$-glutamyltransferase.

on archival tumor tissue. We first examined VEGFR1, 2, and 3 expressions and found no correlation between PFS and expression levels (Figure $2 \mathrm{~A}-\mathrm{C}$ ). Since PDGFR $\alpha / \beta$ has also has been implicated in sarcoma response, we then tested PDGFR $\alpha / \beta$ expression and again demonstrated no correlation between expression and response (Figure 2D and E). Finally, given tumor heterogeneity, we attempted to associate response with circulating levels of VEGF, PDGF $\alpha$, PDGF $\beta$ and FGF2. Again we found no correlation between PFS and circulating ligands (Figure 2F-I).

\section{Discussion}

The primary objective of this study was to assess the antitumor efficacy of tivozanib in patients with metastatic STS. This was a histologically heterogeneous cohort with undifferentiated sarcomas comprising $16 \%$ of the patients and up $20 \%$ with undefined tumor histology. This trial enrolled a heavily pretreated population with progressive STS, in which $46 \%$ of the patients had a least 3 lines of previous systemic therapy. Tivozanib was well tolerated with manageable grade 3 toxicities, most common being hypertension (22.4\%) and reduced ejection fraction (8.6\%). The toxicity profile was in line with expected results from studies in renal cell carcinomas $[22,23]$.

The estimated 16-week PFS rate in our study was 36.4\% (95\% CI 23.7-49.1) and the study met its primary endpoint for
Table 3. Frequency of treatment related all grade 3-4 AEs

Toxicity event

\begin{tabular}{|c|c|c|c|}
\hline \multicolumn{2}{|c|}{ Grade 3} & \multicolumn{2}{|c|}{ Grade 4} \\
\hline$n$ & $\%$ & $n$ & $\%$ \\
\hline 13 & 22.4 & 1 & 1.7 \\
\hline 5 & 8.6 & 0 & 0.0 \\
\hline 4 & 6.9 & 0 & 0.0 \\
\hline 1 & 1.7 & 0 & 0.0 \\
\hline 1 & 1.7 & 0 & 0.0 \\
\hline 1 & 1.7 & 0 & 0.0 \\
\hline 1 & 1.7 & 0 & 0.0 \\
\hline 1 & 1.7 & 0 & 0.0 \\
\hline 1 & 1.7 & 0 & 0.0 \\
\hline 1 & 1.7 & 0 & 0.0 \\
\hline 1 & 1.7 & 0 & 0.0 \\
\hline 1 & 1.7 & 0 & 0.0 \\
\hline 1 & 1.7 & 0 & 0.0 \\
\hline 1 & 1.7 & 0 & 0.0 \\
\hline 1 & 1.7 & 0 & 0.0 \\
\hline 1 & 1.7 & 0 & 0.0 \\
\hline 1 & 1.7 & 0 & 0.0 \\
\hline 1 & 1.7 & 0 & 0.0 \\
\hline 1 & 1.7 & 0 & 0.0 \\
\hline
\end{tabular}

Hypertension

Ejection fraction decreased

Fatigue

Anemia

Stomach pain

Lower gastrointestinal hemorrhage

Gait disturbance

Alanine aminotransferase increased

Alkaline phosphatase increased

Aspartate aminotransferase increased

Lymphocyte count decreased

Platelet count decreased

Weight loss

Hypokalemia

Hypophosphatemia

Myalgia

Reversible posterior

leukoencephalopathy syndrome

Urinary retention

Pneumothorax

AEs, adverse events.

rejection of null hypothesis. PFS rate was chosen as primary endpoint of this study given its known positive correlation between PFS rate and OS improvement in STSs phase II trials [24]. We also considered the 16-week time point would discriminate between drug efficacy and slow disease growing disease when compared to earlier assessments of disease control.

Results of the EORTC database analysis of 380 cases of previously treated STSs showed that a 12 -week PFS rate of $39 \%$ in phase II trials indicate an active drug [21]. The corollary to this paradigm is that tivozanib showed promising antitumor activity in our study with a Kaplan-Meier curve estimated 12-week PFS rate of $52.7 \%$ (95\% CI 39.6-65.8). Furthermore, the phase II study of pazopanib in pretreated patients with STSs showed a 12week PFS rate of approximately $40 \%$ in STSs prior to meeting its primary end point of PFS improvement in randomized phase III trial (hazard ratio of $0.31,95 \%$ CI $0.24-0.40 ; P<0.0001$ ) [14, 25]. With respect to median PFS, the PALETTE trial showed a median progression-free survival of 4-6 months (95\% CI 3.74.8) for pazopanib [14], while in our study the median PFS of tivozanib was 3.5 months, (95\% CI 1.8-3). Similarly, other phase II studies with TKIs (sorafenib, sunitinib and cediranib) showed promising antitumor activity in STSs indicating kinase signaling abrogation correlates with improvement of outcomes [26-28]. It is important to highlight that in exploratory analysis patients with history of previous exposure to anti-VEGF treatment derived benefit from tivozanib treatment and patients progressing on anti-angiogenic treatment can be considered for tivozanib treatment. This benefit may be a reflection of the multiple targets being inhibited by these individual agents. 
A

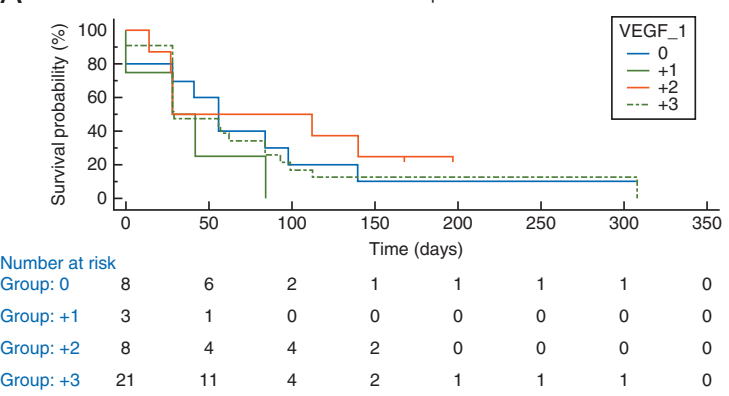

C

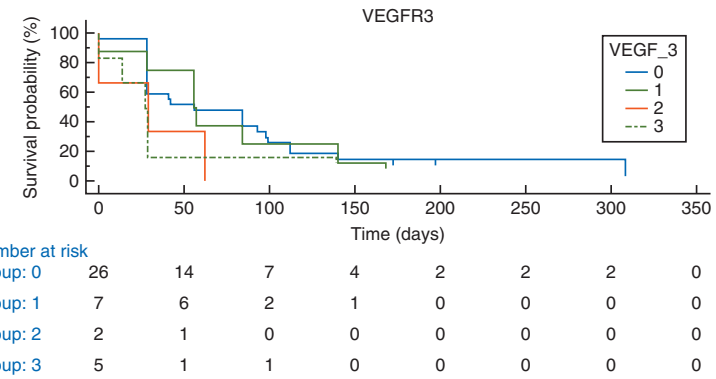

$\mathrm{E}$

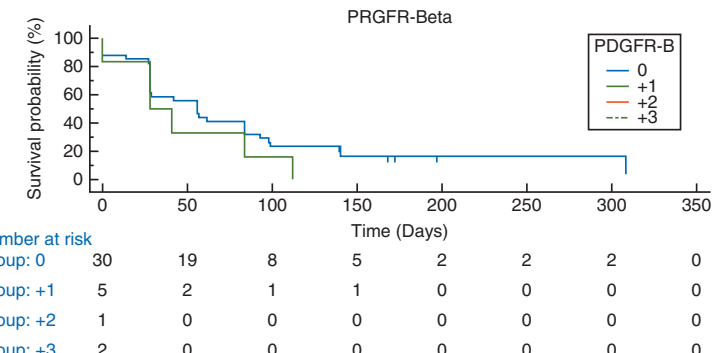

B

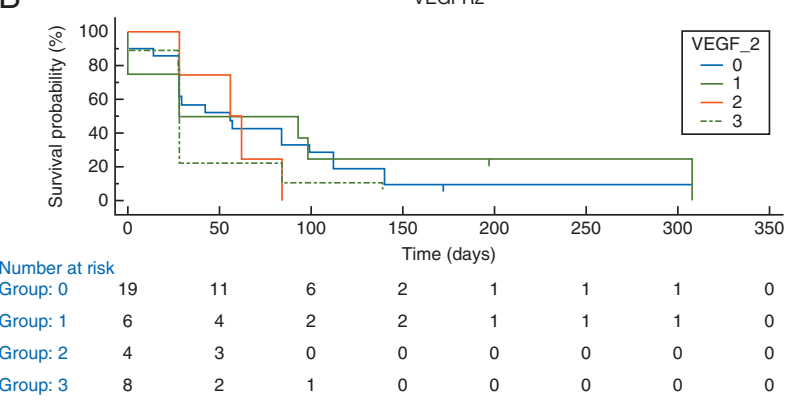

D

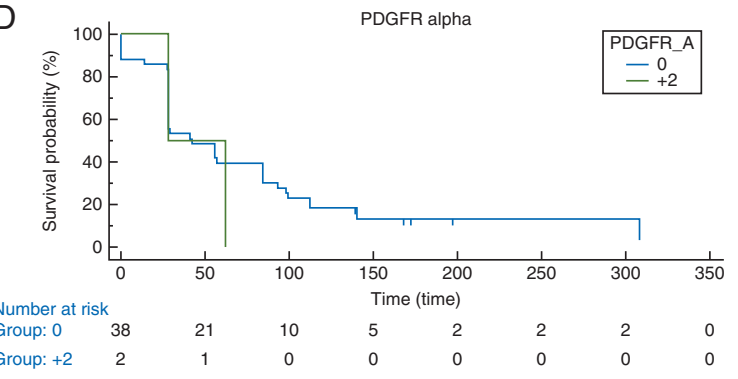

$\mathrm{F}$

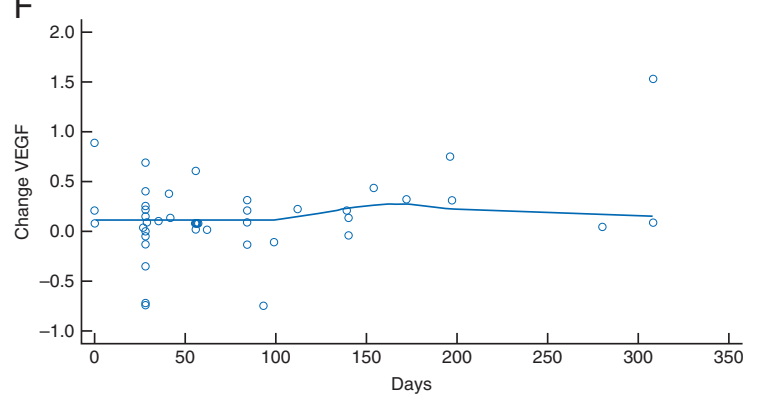

G

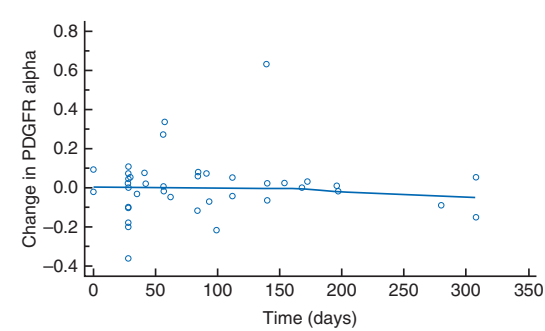

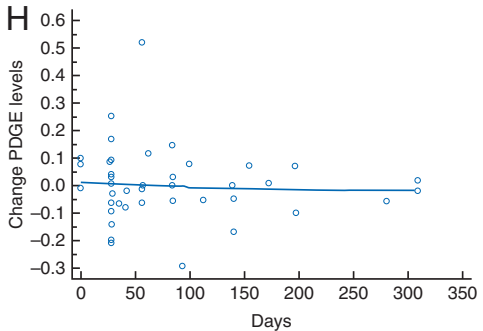

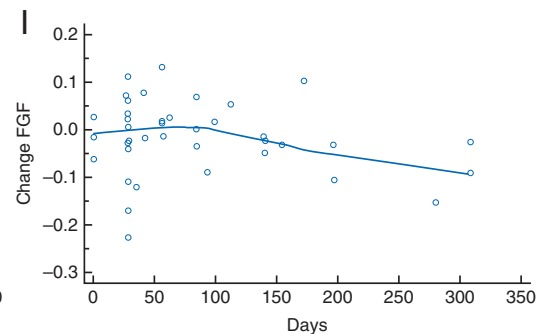

Figure 2. Kalan-Meier curves demonstrating no correlation between expression of (A) VEGFR1 (B) VEGFR2 (C) VEGFR3 (D) PDGFRa (E) PDGFRb with days on trial. The changes in circulating expression between day 30 minus day 1 of (F) VEGF (G) PDGFRa (H) PDGFRb (E) FGF2 did not correlate with time on trial.

The correlative analysis demonstrated no association between response expression o9f VEGFR 1,2,3, PDGFR $\alpha / \beta$ as seen by IHC, or circulating VEGF, PDGF or FGF levels. With over 90 receptor tyrosine kinases and the promiscuity of most TKIs across the kinome, our finding that tivozanib response did not correlate with the expected targets of VEGFR 1,2 or 3 is not all that surprising. Given the large number of subtypes of sarcoma $(75+)$, it would be unusual for all the subtypes of sarcoma to be dependent on the same TKIs. Additionally, tivozanib may be inhibiting a key kinase that has not been clearly implicated in STS at this time. Alternatively, there are possible limitations to the use of archival tissue and IHC for the detection of a biomarker which could alter the interpretation of the IHC presented as correlatives [29-31]. First, protein expression, as detected by IHC, must reach a threshold of expression for detection that may well above that needed for function, as there are interlaboratory variation that seen with the same antibodies. Second, even with modern antigen retrieval methods, cross linking by different methods at different sites may mask signal. Finally, even with well accepted antibodies, we are unable to validate that they are hitting their protein of interest in fixed section due to crossreactivity. A good example of this is from the Olartumab trial where the first antibody used was not specific to PDGFR $\alpha$, and upon replacement and reinterprestation with a second antibody there was still no correlation between PDGFR $\alpha$ expression and outcomes \{Tap, \#2295\}. Future developments of TKIs in STS depend on either elucidating the oncogenic TK or broadening the number of tyrosine kinases that are inhibited by a molecule without increasing toxicity. Further research in this area is needed. 
In conclusion, tivozanib demonstrated antitumor activity in patients with metastatic refractory STS and was well tolerated. Henceforth, further clinical trials are needed to assess the definitive clinical activity of tivozanib. For instance, a maintenance design trial after metastatectomy or completion of standard chemotherapy would allow randomization to placebo. Currently no TKIs are approved for the treatment of liposarcoma. This study showed SD as the best result in 3 of the 4 liposarcoma patients, which may merit further exploration in an expansion cohort. Finally, looking at novel combinations with this agent is of particular interest inasmuch as it could lead to significant improvement in outcomes.

\section{Acknowledgements}

We would like to thank the Northwestern Memorial Foundation DeBoer Family Research Initiative for funding the correlatives in this trial.

\section{Funding}

Supported by AVEO Oncology, One Broadway, 14th Floor Cambridge, MA 02142 USA and Northwestern Memorial Foundation, DeBoer Family Sarcoma Research Grant Initiative (no grant number is applicable).

\section{Disclosure}

MA: Consulting or Advisory Role: EMD Serono, Janssen Pharmaceuticals, Eisai, Novartis. Speaker's Bureau: Novartis, GlaxoSmithKline. Mohammed Milhem: Consulting or Advisory Role: EMD Serono, Amgen, Eisai, Novartis, Genentech, BMS. Scott Okuno: Consulting or Advisory Role: EMD Serono. Brian Van Tine: Consulting or Advisory Role: Advaxis, GlaxoSmithKline, EMD Serono, Novartis, Lilly/ImClone, Johnson \& Johnson, Caris Life Sciences, DFINE, Threshold Pharmaceuticals, Karyopharm Therapeutics. Speaker's Bureau: GlaxoSmithKline, Caris Life Sciences, DFINE, Johnson \& Johnson. Research Funding: Polaris, Morphotek. All remaining authors have declared no conflicts of interest.

\section{References}

1. Siegel RL, Miller KD, Jemal A. Cancer statistics, 2015. CA Cancer J Clin 2015; 65: 5-29.

2. Santoro A, Tursz T, Mouridsen $\mathrm{H}$ et al. Doxorubicin versus CYVADIC versus doxorubicin plus ifosfamide in first-line treatment of advanced soft tissue sarcomas: a randomized study of the European organization for research and treatment of cancer soft tissue and bone sarcoma group. J Clin Oncol 1995; 13: 1537-1545.

3. Lorigan P, Verweij J, Papai Z et al. Phase III trial of two investigational schedules of ifosfamide compared with standard-dose doxorubicin in advanced or metastatic soft tissue sarcoma: a European organisation for research and treatment of cancer soft tissue and bone sarcoma group study. J Clin Oncol 2007; 25: 3144-3150.

4. Ryan CW, Schoffski P, Merimsky O et al. PICASSO III: A Phase III, Placebo-Controlled Study of Doxorubicin With or Without
Palifosfamide in Patients With Metastatic Soft Tissue Sarcoma. J Clin Oncol. 2016 Sep 12; pii: JCO676684.

5. Tap WD, Jones RL, Van Tine BA et al. Olaratumab and doxorubicin versus doxorubicin alone for treatment of soft-tissue sarcoma: an open-label phase $1 \mathrm{~b}$ and randomised phase 2 trial. Lancet. 2016; 388(10043): 48897. doi: 10.1016/S0140-6736(16)30587-6.

6. Folkman J. What is the evidence that tumors are angiogenesis dependent? J Natl Cancer Inst 1990; 82: 4-6.

7. Folkman J. Role of angiogenesis in tumor growth and metastasis. Semin Oncol 2002; 29: 15-18.

8. Arita S, Kikkawa F, Kajiyama $\mathrm{H}$ et al. Prognostic importance of vascular endothelial growth factor and its receptors in the uterine sarcoma. Int J Gynecol Cancer 2005; 15: 329-336.

9. Pakos EE, Goussia AC, Tsekeris PG et al. Expression of vascular endothelial growth factor and its receptor, KDR/Flk-1, in soft tissue sarcomas. Anticancer Res 2005; 25: 3591-3596.

10. Yoon SS, Segal NH, Olshen AB et al. Circulating angiogenic factor levels correlate with extent of disease and risk of recurrence in patients with soft tissue sarcoma. Ann Oncol 2004; 15: 1261-1266.

11. Hayes AJ, Mostyn-Jones A, Koban MU et al. Serum vascular endothelial growth factor as a tumour marker in soft tissue sarcoma. Br J Surg 2004; 91: 242-247.

12. Zhang L, Hannay JA, Liu J et al. Vascular endothelial growth factor overexpression by soft tissue sarcoma cells: implications for tumor growth, metastasis, and chemoresistance. Cancer Res 2006; 66: 8770-8778.

13. Schutz FA, Choueiri TK, Sternberg CN. Pazopanib: clinical development of a potent anti-angiogenic drug. Crit Rev Oncol Hematol 2011; 77: $163-171$.

14. van der Graaf WT, Blay JY, Chawla SP et al. Pazopanib for metastatic soft-tissue sarcoma (PALETTE): a randomised, double-blind, placebocontrolled phase 3 trial. Lancet 2012; 379: 1879-1886.

15. Nakamura K, Taguchi E, Miura T et al. KRN951, a highly potent inhibitor of vascular endothelial growth factor receptor tyrosine kinases, has antitumor activities and affects functional vascular properties. Cancer Res 2006; 66: 9134-9142.

16. Jamil MO, Hathaway A, Mehta A. Tivozanib: status of development. Curr Oncol Rep 2015; 17: 24.

17. Mayer EL, Scheulen ME, Beckman J et al. A Phase I dose-escalation study of the VEGFR inhibitor tivozanib hydrochloride with weekly paclitaxel in metastatic breast cancer. Breast Cancer Res Treat 2013; 140: 331-339.

18. Fishman MN, Srinivas S, Hauke RJ et al. Phase Ib study of tivozanib (AV-951) in combination with temsirolimus in patients with renal cell carcinoma. Eur J Cancer 2013; 49: 2841-2850.

19. Eisenhauer EA, Therasse P, Bogaerts J et al. New response evaluation criteria in solid tumours: revised RECIST guideline (version 1.1). Eur J Cancer 2009; 45: 228-247.

20. Simon R. Optimal two-stage designs for phase II clinical trials. Control Clin Trials 1989; 10: 1-10.

21. Van Glabbeke M, Verweij J, Judson I, Nielsen OS. Progression-free rate as the principal end-point for phase II trials in soft-tissue sarcomas. Eur J Cancer 2002; 38: 543-549.

22. Nosov DA, Esteves B, Lipatov ON et al. Antitumor activity and safety of tivozanib (AV-951) in a phase II randomized discontinuation trial in patients with renal cell carcinoma. J Clin Oncol 2012; 30: 1678-1685.

23. Motzer RJ, Nosov D, Eisen T et al. Tivozanib versus sorafenib as initial targeted therapy for patients with metastatic renal cell carcinoma: results from a phase III trial. J Clin Oncol 2013; 31: 3791-3799.

24. Penel N, Cousin S, Duhamel A, Kramar A. Activity endpoints reported in soft tissue sarcoma phase II trials: quality of reported endpoints and correlation with overall survival. Crit Rev Oncol Hematol 2013; 88: 309-317.

25. Sleijfer S, Ray-Coquard I, Papai Z et al. Pazopanib, a multikinase angiogenesis inhibitor, in patients with relapsed or refractory advanced soft tissue sarcoma: a phase II study from the European organisation for research and treatment of cancer-soft tissue and bone sarcoma group (EORTC study 62043). J Clin Oncol 2009; 27: 3126-3132. 
26. Kummar S, Allen D, Monks A et al. Cediranib for metastatic alveolar soft part sarcoma. J Clin Oncol 2013; 31: 2296-2302.

27. Maki RG, D'Adamo DR, Keohan ML et al. Phase II study of sorafenib in patients with metastatic or recurrent sarcomas. J Clin Oncol 2009; 27: 3133-3140.

28. Mahmood ST, Agresta S, Vigil CE et al. Phase II study of sunitinib malate, a multitargeted tyrosine kinase inhibitor in patients with relapsed or refractory soft tissue sarcomas. Focus on three prevalent histologies: leiomyosarcoma, liposarcoma and malignant fibrous histiocytoma. Int J Cancer 2011; 129: 1963-1969.
29. Chames P, Van Regenmortel M, Weiss E, Baty D. Therapeutic antibodies: successes, limitations and hopes for the future. Br J Pharmacol 2009; 157: 220-233.

30. Fitzgibbons PL, Bradley LA, Fatheree LA et al. Principles of analytic validation of immunohistochemical assays: guideline from the college of American pathologists pathology and laboratory quality center. Arch Pathol Lab Med 138: 1432-1443.

31. Ramos-Vara JA, Kiupel M, Baszler $\mathrm{T}$ et al. Suggested Guidelines for Immunohistochemical Techniques in Veterinary Diagnostic Laboratories. J Vet Diagn Invest 2008; 20: 393-413. 